\title{
Optimal boundary control problems related to high-lift configurations
}

\author{
Christian John, Bernd R. Noack, Michael Schlegel, Fredi Tröltzsch, and \\ Daniel Wachsmuth
}

\begin{abstract}
We investigate two control problems related to the aerodynamic optimization of flows around airfoils in high-lift configurations. The first issue is the steady state maximization of lift subject to restrictions on the drag. This leads to a boundary control problem for the 2D stationary Navier-Stokes equations with constrained controls functions belonging to $\mathbf{L}^{2}(\Gamma)$ under an integral state constraint. We derive optimality conditions and treat the problem numerically by direct solution of the associated nonsmooth optimality system. The second part is based on a $k-\omega$ WILCOX98 turbulence model. To deal with the curse of dimension, we discuss a reduced-order model by adapting a small system of ODEs to solutions computed with the full model.
\end{abstract}

Keywords. Optimal boundary control, Navier-Stokes equations, lift maximization, reduced-order model, turbulence model.

Christian John, Fredi Tröltzsch

Technische Universität Berlin, Institute of Mathematics, Strasse des 17. Juni 136, 10623 Berlin, Germany, e-mail: john@math.tu-berlin.de

Bernd R. Noack, Michael Schlegel

Technische Universität Berlin, MB1, Strasse des 17. Juni 135, 10623 Berlin, Germany, e-mail: michael.schlegel@tu-berlin.de

Daniel Wachsmuth

Johann Radon Institute for Computational and Applied Mathematics (RICAM), Austrian Academy of Sciences, Altenbergerstrasse 69, A-4040 Linz, Austria, e-mail: daniel.wachsmuth@ricam.oeaw.ac.at 


\section{Introduction}

We study some questions of numerical analysis for optimal boundary control problems to optimize the flow around airfoils. The associated background of applications in fluid mechanics, active separation control, was subject of various papers written from an engineering point of view. We only mention [5, 6, 26, 27, 28, 36], whose considerations are close to our setting.

Mathematically, this class of problems belongs to the field of optimal control of Navier-Stokes equations. Starting with the paper [1], an almost countless number of associated contributions has been published. In particular, we refer to $[8,9,11,12$, $13,14,32,34]$. Optimal flow control problems with state constraints were studied in $[10,24,25]$.

Our paper deals with two problems, both related to high-lift configurations, where the lift is increased by suction or blowing of flow on part of a wing. In the first part, we consider a steady state optimal boundary control problem for the twodimensional Navier-Stokes equations with low Reynolds number. Here, a linearquadratic integral functional expressing the lift is to be maximized under an integral state constraint on the drag. The control acts on a part of the boundary of the domain and is taken from the space $L^{2}(\Gamma)$. This raises some problems of regularity which have to be solved. After a suitable transformation of the problem, we derive first-order necessary optimality conditions. The associated nonsmooth first-order optimality system of two coupled Navier-Stokes equations is solved numerically following a method suggested by Neitzel et al. [21].

The second part of the paper deals with a nonstationary problem that accounts also for turbulence. Here, the flow is computed on the basis of a $k-\omega$ WILCOX98 model including the nonstationary Navier-Stokes equations. The curse of dimension leads to very large computing times so that a model reduction is a method of choice. To this aim, many authors have considered proper orthogonal decomposition (POD). We mention e.g. [2, 18, 19, 35]. A reduced-order model (ROM) is considered in Section 3 due to [20] and [22]. The numerical application of this technique is explained in Section 3 and first test examples are presented.

\section{A stationary optimal boundary control problem}

\subsection{Definition of the problem}

In this section, we discuss the stationary optimal control problem to maximize the lift under a restriction on the drag. Here, we assume a low Reynolds number so that we avoid the discussion of turbulence. The computational domain, i.e. the domain of the flow field is denoted by $\Omega \subset \mathbb{R}^{n}, n=2,3$, cf. Figure 1 . Its boundary $\Gamma=\partial \Omega$ covers in particular the boundaries of the wings shown in the figure. These parts of the boundary, denoted by $\Gamma_{w}$, are assumed to be sufficiently smooth. The boundary 
$\Gamma$ is the union of $m$ connected components, $\Gamma=\bigcup_{j=1}^{m} \Gamma_{j}$. We assume for simplicity that the control $g$ acts on a subdomain of $\Gamma_{w}$ denoted by $\Gamma_{c}$. The set $\Gamma_{c}$ stands for the location of the slit for suction and blowing and we avoid a precise modeling of the associated jet. The velocity field $u$ around the wing is obtained as the solution of the stationary incompressible Navier-Stokes equations

$$
\begin{aligned}
& -v \Delta u+(u \cdot \nabla) u+\nabla p=0 \quad \text { in } \Omega \\
& \operatorname{div} \mathrm{u}=0 \quad \text { in } \Omega \\
& u=g \quad \text { on } \Gamma_{c}, \\
& u=0 \quad \text { on } \Gamma \backslash \Gamma_{c} \text {, }
\end{aligned}
$$

where $p$ denotes the pressure associated with the flow. The parameter $v=1 / \operatorname{Re}$ is related to the viscosity. The admissible controls are taken from the set $G_{a d} \subset L^{2}(\Gamma)$ defined by

$$
G_{a d}:=\left\{g \in \mathbf{H}^{0}(\Gamma): g(x) \in G \text { a.e. on } \Gamma_{c}\right\},
$$

where $G$ is a bounded, convex, closed, and non-empty subset of $\mathbb{R}^{n}$. For instance, $G$ can be given by lower and upper bounds, $G=\left[G_{a}, G_{b}\right]$. We also assume $0 \in G$ to have the option of turning off the control. The space $\mathbf{H}^{0}(\Gamma)$ consists of all functions $h \in H^{0}(\Gamma)$ with $\int_{\Gamma_{j}} h \cdot \mathfrak{n} d \gamma=0$ for all $j=1, \cdots, m$. For a discussion of the used function spaces and such convex control constraints, we refer to [16] and [33].

The force on the wing in a direction $\mathbf{e}$ is given by the boundary integral

$$
F=\int_{\Gamma_{w}}\left(v \partial_{\mathfrak{n}} u-p \mathfrak{n}\right) \cdot \mathbf{e} \mathrm{d} \gamma
$$

where $\mathfrak{n}$ is the outward normal vector and we write for short $\partial_{\mathfrak{n}} u:=\partial u / \partial \mathfrak{n}$. Let the vectors $\mathbf{e}_{l}$ and $\mathbf{e}_{d}$ indicate the directions of lift and drag. Now, we are able to formulate our optimal control problem:

Find a control $g \in G_{a d}$ that maximizes the lift, i.e. minimizes the negative lift,

$$
\min F_{l}(u):=-\int_{\Gamma_{w}}\left(v \partial_{\mathfrak{n}} u-p \mathfrak{n}\right) \cdot \mathbf{e}_{l} \mathrm{~d} \gamma
$$

subject to (1) and, with some given constant $d_{0}$, to the integral state (drag) constraint

$$
F_{d}(u)=\int_{\Gamma_{w}}\left(v \partial_{\mathfrak{n}} u-p \mathfrak{n}\right) \cdot \mathbf{e}_{d} \mathrm{~d} \gamma \leq \mathrm{d}_{0} .
$$

\subsection{Well-posedness and optimality conditions}

The analysis of the optimal control problem (1)-(4) was developed in [16]; the associated assumptions are satisfied by our setting. Below, we state the main conclusions for (1)-(4). The solutions of the state equations (1) are defined in the very weak sense 
and it follows that for every control function $g \in \mathbf{H}^{0}(\Gamma)$ there exists a very weak solution $u \in \mathbf{Y}:=\mathbf{L}^{2 n /(n-1)}(\Omega)$ of (1), see [16], Section 2. For this result to hold, it is essential to have $\int_{\Gamma_{i}} g \cdot n \mathrm{~d} \gamma=0$ for all connected components $\Gamma_{i}$ of $\Gamma$. If $g$ is small compared to $v$, then the solution $u$ is unique.

The assumption of non-singularity implies that the Navier-Stokes equation is uniquely solvable in a neighborhood of the reference control and state, we refer to [7, Theorem 2.5] Notice that our optimal control problem is nonlinear and hence belongs to the field of nonconvex optimization. Therefore, we have to deal with locally optimal solutions. In what follows, $\bar{g}$ is a locally optimal reference control and $\bar{u}$ is the associated state. A pair $(\bar{u}, \bar{g}) \in \mathbf{Y} \times \mathbf{H}^{0}(\Gamma)$ is called non-singular, if the Navier-Stokes equations linearized at $\bar{u}$ admit a unique very weak solution $u \in \mathbf{Y}$ for all boundary data $g \in \mathbf{H}^{0}(\Gamma)$ and all distributed data $f \in \mathbf{H}^{-1}(\Omega)$ and the solution mapping $(g, f) \mapsto u$ is linear and continuous. This condition is fulfilled for small $\bar{u},[23$, Lemma B.1].

In the cost functional $F_{l}$ defined in (3) and $F_{d}$ defined in (4), the normal derivative $\partial_{\mathfrak{n}} u$ appears; therefore we need $\partial_{\mathfrak{n}} u \in L^{1}(\Gamma)$. For Dirichlet boundary data $g \in L^{2}(\Gamma)$ this is not true, hence $F_{l}$ and $F_{d}$ were reformulated in [16], Subsection 2.4, to $f_{l}(u, g)$ and $f_{d}(u, g)$ defined as

$$
f_{i}(u, g)=\int_{\Omega}\left(v u \cdot \Delta \varphi_{i}+(u \cdot \nabla) \varphi_{i} u\right) \mathrm{dx}-\int_{\Gamma}\left(v \mathrm{~g} \partial_{\mathfrak{n}} \varphi_{\mathrm{i}}+(\mathrm{u} \cdot \mathrm{n})\left(\varphi_{\mathrm{i}} \cdot \mathrm{g}\right)\right) \mathrm{d} \gamma,
$$

$i \in\{d, l\}$, where $u \in \mathbf{Y}$ is regular enough. This transformation is obtained by multiplying (1) with some $\varphi \in \mathbf{H}^{2}(\Omega)$ and integrating twice partially so that $F_{i}(u)=$ $f_{i}(u, g), i \in\{d, l\}$. To show the existence of an optimal solution, we have the problem that the cost functional is not bounded from below, because the solution of the state equation is not necessarily unique for large data. To resolve this problem, we modify the original cost functional to

$$
\tilde{J}(u, g):=m\left(-f_{l}(u, g)\right)+\frac{\alpha_{m}}{2}\|u\|_{\mathbf{H}^{1 / 2}}^{2}+\frac{\alpha}{2}\|g\|_{\mathbf{H}^{0}}^{2} .
$$

where $\alpha_{m}$ is a small positive parameter. The function $m: \mathbb{R} \rightarrow \mathbb{R}$ is assumed to be continuous, monotone increasing, and bounded from below. We have to modify also the control constraints by

$$
\tilde{G}_{a d} \subset\left\{u \in G_{a d}: \int_{\Gamma_{w}}(g \cdot n)\left(\varphi_{i} \cdot g\right) \mathrm{d} \gamma=0, \mathrm{i} \in\{\mathrm{d}, 1\}\right\} .
$$

If there is an admissible pair $(\tilde{u}, \tilde{g}) \in \mathbf{H}^{1 / 2}(\Omega) \times \tilde{G}_{a d}$ satisfying all constraints, i.e. (1), (3) and (4), where $f_{l}$ and $f_{d}$ are substituted for $F_{l}$ and $F_{d}$, respectively, then the modified problem possesses at least one solution. To avoid specific numerical difficulties and problems with traces and low state regularity, we confine ourselves to a finite-dimensional control set $G_{a d, q}:=\left\{g \in \mathbf{H}^{1 / 2}(\Gamma): g=\sum_{i=1}^{l} q_{i} e_{i}, q \in Q\right\}$ with $Q \in \mathbb{R}^{l}$, [[16], Section 4]. Due to $g \in \mathbf{H}^{1 / 2}(\Gamma)$, the associated state $u$ belongs to $\mathbf{H}^{1}(\Omega)$, so we are able to reformulate $F_{l}$ and $F_{d}$ to: 


$$
\hat{f}_{i}(u):=-\int_{\Omega}\left(v \nabla u \cdot \nabla \varphi_{i}+(u \cdot \nabla) u \varphi_{i}\right) \mathrm{dx}, \mathrm{i} \in\{\mathrm{d}, 1\} .
$$

Then $\hat{f}_{i}$ is twice continuously Fréchet differentiable from $\mathbf{H}^{1}(\Omega)$ to $\mathbb{R}$ and it holds $\hat{f}_{i}(u)=f_{i}(u, g)=F_{i}, i \in\{d, l\}$, for smooth states $u$ associated to controls $g$. The following theorem holds true:

Theorem 1. Let $(\bar{u}, \bar{g}) \in \boldsymbol{Y} \times G_{a d}$ a non-singular locally optimal solution for the modified optimal control problem. Assume that there exist $\tilde{g}$ close to $\bar{g}$ satisfying a the standard linearized Slater condition. Then there is a Lagrange multiplier $\xi \geq$ 0 associated with the integral state constraint (4), an adjoint state $\lambda \in \boldsymbol{H}_{0}^{1}(\Omega) \cap$ $W^{2, r}(\Omega)^{n}$, and an associated adjoint pressure $\pi \in W^{1, r}(\Omega)$, for all $r \in[2, \infty)$, such that $(\lambda, \pi)$ is the weak solution of the adjoint equation

$$
\begin{aligned}
-v \Delta \lambda+(\nabla \bar{u})^{T} \lambda-(\bar{u} \cdot \nabla) \lambda+\nabla \pi & =0 & & \text { in } \Omega \\
\operatorname{div} \lambda & =0 & & \text { in } \Omega \\
\lambda & =e_{l}-\xi e_{d} & & \text { on } \Gamma_{w} \\
\lambda & =0 & & \text { on } \Gamma \backslash \Gamma_{w}
\end{aligned}
$$

and the following conditions are satisfied: The variational inequality

$$
\int_{\Gamma_{c}}\left(\alpha \bar{g}-\left(v \partial_{\mathfrak{n}} \lambda-\pi \mathfrak{n}\right)\right) \cdot(g-\bar{g}) \geq 0 \quad \forall g \in G_{a d, q}
$$

and the complementarity condition

$$
\xi\left(\hat{f}_{d}(\bar{u})-d_{0}\right)=0, \xi \geq 0, \hat{f}_{d}(\bar{u}) \leq d_{0} .
$$

\subsection{Numerical solution}

We handle the optimal control above numerically by direct solution of the optimality system that follows from Theorem 1 and is stated below, see also [16], Section 6. Our computations are based on the following setting: The computational domain is related to a 2D generic high-lift configuration and is shown in Figure 1.

The inflow $u_{\infty}$ acts through an inhomogeneous Dirichlet boundary condition at the inflow boundary $\Gamma_{i n}$ The control boundary $\Gamma_{c}$ was modelled by a nonhomogeneous Dirichlet condition, where the (bounded) suction and blowing occurs by a small slot in the flap. A no-slip boundary condition, i.e. homogeneous Dirichlet condition, was used for the remaining airfoil $\Gamma_{w}$ and the wall boundaries $\Gamma_{w a l l}$. At the outflow boundary $\Gamma_{\text {out }}$, we prescribe a so called "do nothing" condition: $v \partial_{\mathfrak{n}} u-p \mathfrak{n}=0$. For more details of the configuration see the technical report [6].

Thanks to the do nothing boundary condition, we can abandon the constraint $\int_{\Gamma_{j}} u \cdot n \mathrm{~d} \gamma=0$, which was incorporated to guarantee existence of divergence free solutions. With this simplification, the variational inequality and the complementarity 


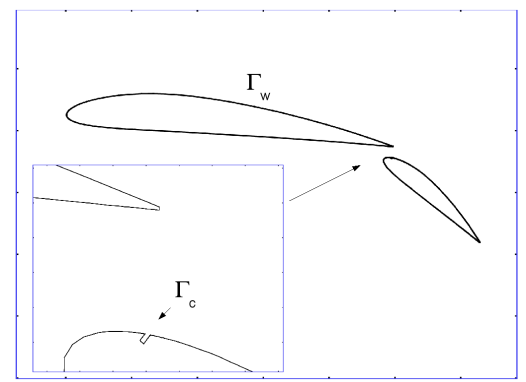

Fig. 1 The generic high-lift configuration where the slit for the control function is implemented on the flap.

condition in the optimality system given by Theorem 1 are equivalent to

$$
\bar{g}=\mathbb{P}_{\mathrm{G}}\left\{1 / \alpha\left(v \partial_{\mathfrak{n}} \lambda-\pi \mathfrak{n}\right)\right\} \text { in } \Gamma_{\mathrm{c}}
$$

and

$$
\xi=\max \left(0, \xi+\hat{f}_{d}(\bar{u})-d_{0}\right),
$$

where $\mathbb{P}_{G}$ is the projection of $\mathbb{R}^{2}$ to $G$. For $G=\left[G_{a}, G_{b}\right], \mathbb{P}$ is defined by $\mathbb{P}_{\left[G_{a}, G_{b}\right]}\{g\}:=$ $\min \left\{G_{b}, \max \left\{G_{a}, g\right\}\right\}$. This projection permits to eliminate the control variable. Then we have to solve the following nonsmooth optimality system consisting of the state equation, the equation for the Lagrange multiplier $\xi$ and the associated adjoint equation:

$$
\begin{aligned}
-v \Delta u+(u \cdot \nabla) u+\nabla p & =0 & & \text { in } \Omega, \\
\operatorname{div} u & =0 & & \text { in } \Omega, \\
u & =\mathbb{P}_{\mathrm{G}}\left(1 / \alpha\left(v \partial_{\mathfrak{n}} \lambda-\pi \mathfrak{n}\right)\right) & & \text { on } \Gamma_{c}, \\
u & =0 & & \text { on } \Gamma_{\text {wall }} \cup \Gamma_{w} \backslash \Gamma_{c}, \\
u & =u_{\infty} & & \text { on } \Gamma_{i n}, \\
v \partial_{\mathfrak{n}} u-p \mathfrak{n} & =0 & & \text { on } \Gamma_{\text {out }}, \\
-v \Delta \lambda+(\nabla u)^{T} \lambda-(u \cdot \nabla) \lambda+\nabla \pi & =0 & & \\
\operatorname{div} \lambda & =0 & & \text { in } \Omega, \\
\lambda & =e_{l}-\xi e_{d} & & \text { in } \Omega, \\
\lambda & =0 & & \text { on } \Gamma_{w}, \\
\xi \partial_{\mathfrak{n}} \lambda-\pi \mathfrak{n}+(u \cdot \mathfrak{n}) \lambda & =0 & & \text { on } \Gamma_{\text {in }} \cup \Gamma_{\text {wall }}, \\
\xi & =\max \left(0, \xi+\hat{f}_{d}(u)-d_{0}\right) . & &
\end{aligned}
$$


We used the commercial Finite-Element code COMSOL Multiphysics with a damped Newton method to solve the nonlinear system. The partial differential equations were discretized by Taylor-Hood finite elements. The Reynolds number given by $R e=106.25$ is based on the chord length $L_{r e f}=1.275$ and the free stream velocity $u_{\infty}=1$. For the uncontrolled problem, we obtained a lift coefficient of $C_{a}=$ $F_{A} /\left(0.5 u_{\infty}^{2} L_{r e f}\right)=1.299$ and a drag coefficient of $C_{d}=D_{A} /\left(0.5 u_{\infty}^{2} L_{r e f}\right)=0.701$, where $F_{A}$ is the resulting lift and $D_{A}$ the drag.
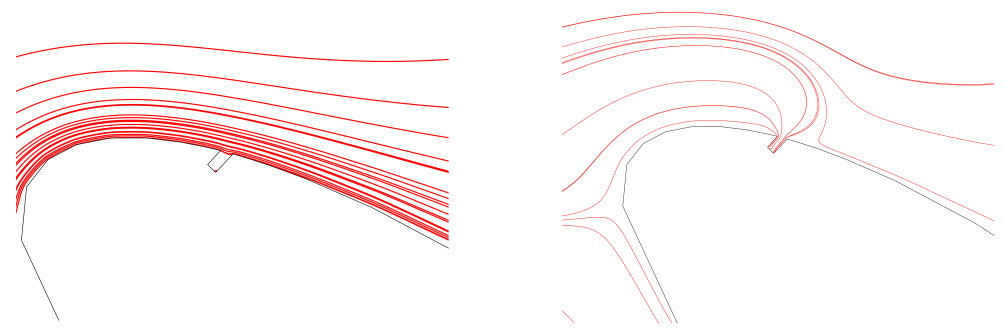

Fig. 2 Velocity field for the uncontrolled (left) and controlled case (right).
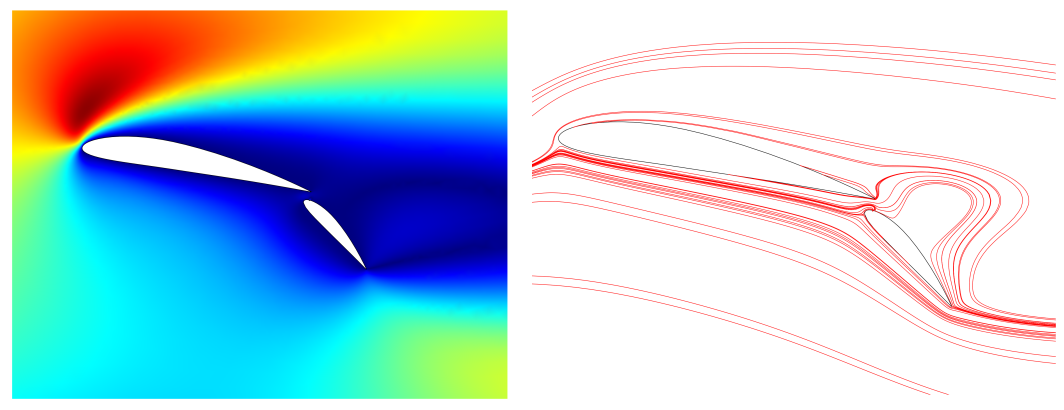

Fig. 3 Controlled case: absolute value of velocity field (left) and streamlines (right).

For the optimization process, we took the control cost parameter $\alpha=0.001$ and the control constraints as box constraints $G=[-1,+1]$, an academic choice to test the solution method. The solution for the case without any drag constraint was maximal possible suction (i.e. $g \equiv-1$ ). The optimized lift is $C_{a}=1.313$ and the associate drag is $C_{d}=0.72$, which is a lift gain of $1.1 \%$. We imposed the upper bound $d_{0}=0.717$ on the drag, which is smaller than 0.72 ; hence this constraint should be active at the solution. In fact, for the computed solution we obtain $C_{d}=0.717$. Due to this restriction, the computed lift $C_{a}=1.3127$ is smaller than for the case without state constraints, but still better than in the uncontrolled situation. 


\section{The nonstationary case}

\subsection{Model reduction}

In contrast to Section 2, we admit in the nonstationary situation high Reynolds numbers and consider a problem closer to the real setting of the high-lift configuration, see e.g. $[6,28,31]$. Here, we have to deal with turbulence, which is simulated by a $k$ $\omega$-WILCOX98 model, we refer to [37]. Moreover, periodic actuation is applied. Due to the high dimension of the discretized equations, the computing times for any forward solution of the model are extremely large so that a mathematical optimization of the periodic actuation is fairly unrealistic. In [6], a generic high-lift configuration was investigated and one forward solution took about 48 hours. In the case of the SCCH configuration, the computation time was nearly twice that number.

Therefore, model reduction is advisable. A widely used method is POD, [2, 15, $18,19]$. In the case of the high-lift configuration, the application of standard POD does not align to the target of robust dynamical least-order models for the real flow. To establish the ROM, the computed POD basis must be inserted as a Galerkin basis in the real WILCOX98 model. The associated implementation is a time consuming task. There were several approaches to deal with these problems, e.g. an extension of POD to data compression of multiple operation points, see [17] for sequential POD or [30] for DPOD. We follow an alternative approach suggested in [20, 22] of a canonical reduction with parameter identification. Here, a very small system of nonlinear ODEs is adapted to the computed flows in the actuated and non-actuated case. This small system is easily tractable by optimization and we prefer to use a model reduction that does not need full information on the original state equations. In this section, we report on first experience in a simplified two-dimensional setting. Our numerical results are promising for future optimization tasks.

\subsection{Reduced-order model (ROM)}

The procedure is as follows: First, for the unactuated system, $N=567$ snapshots $\hat{u}_{i}^{n}(x):=\hat{u}^{n}\left(x, t_{i}\right)$ were determined at equidistant discrete times $t_{i}, i=1, \cdots, N$, all 6 convective time units. Analogously, $N$ snapshots $\hat{u}_{i}^{a}(x):=\hat{u}^{a}\left(x, t_{i}\right), i=1, \cdots, N$, are computed for the actuated system by a URANS simulation with a WILCOX98 turbulence $k$ - $\omega$-model and a Reynolds number of $1.756 \cdot 10^{6}$. The actuation is $g(t)=$ $B \cos \left(\omega^{a} t\right)$, where $B$ is the actuation amplitude, $\omega^{a}=2 \pi S t^{a}$ the actuation phase, and $S t^{a}=f^{a} c / U_{\infty}$ the Strouhal number with actuation frequency $f^{a} ; c=1$ is the cord length of the wing and $U_{\infty}=1$ the incoming flow velocity. We chose a fairly large amplitude $B$ to get significant differences between the frequencies of the operating conditions .

Data analysis. Next, similarly to POD, all snapshots are processed. For this purpose, we consider only the velocity field $u$ in a certain reference domain, where the 
actuation has the main influence on velocity and lift, see Figure 4 . Therefore, the snapshot velocity data are weighted by the size of their area. We select the first two POD modes of the actuated and non-actuated system carrying the highest energy. For further details of POD see e.g. [15].

Filtering. The snapshots $\hat{u}_{i}^{n}$ and $\hat{u}_{i}^{a}$ are given on a time interval $[0, T]$. To guarantee a multiple of the associated wavelengths, we search for the maximal $k^{a}, k^{n} \in \mathbb{N}$ such that the times $T^{n}=k^{n} \omega^{n}$ and $T^{a}=k^{a} \omega^{a}$ fulfill $T^{n}<T$ and $T^{a}<T$. We calculate the phases $\phi^{n}(t), \phi^{a}(t)$ and radii $\tilde{r}^{n}(t), \tilde{r}^{a}(t)$ by

$$
\begin{aligned}
& a_{1}^{n}(t)+i a_{2}^{n}(t)=\tilde{r}^{n}(t) e^{i \phi^{n}(t)}, \\
& a_{1}^{a}(t)+i a_{2}^{a}(t)=\tilde{r}^{a}(t) e^{i \phi^{a}(t)},
\end{aligned}
$$

where $\left(a_{1}^{n}(t), a_{2}^{n}(t)\right)$ and $\left(a_{1}^{a}(t), a_{2}^{a}(t)\right)$ are the first POD mode coefficient pairs of the natural and the actuated case, respectively. To extract the dominant harmonic oscillation from these POD coefficients, fluctuations of both, the radii $\tilde{r}^{n}(t), \tilde{r}^{a}(t)$ and the frequencies $\phi^{n}(t), \phi^{a}(t)$, are neglected. With the average values $\omega^{n}=\overline{\partial_{t} \phi^{n}(t)}$, $\omega^{a}=\overline{\partial_{t} \phi^{a}(t)}$ and $r^{n}=\overline{\tilde{r}^{n}(t)}, r^{a}=\overline{\tilde{r}^{a}(t)}$, we approximate our filtered coefficients $a_{1}(t)+i a_{2}(t)=r^{n} e^{i \omega^{n} t}$ and $a_{1}(t)+i a_{2}(t)=r^{n} e^{i \omega^{n} t}$. The associated filtered modes are determined by

$$
\begin{array}{ll}
u_{i}(x)=\left(\hat{u}^{n}(x, t)-u_{0}^{n}, a_{i}(t)\right)_{T^{n}}:=\frac{1}{T^{n}} \int_{0}^{T^{n}}\left(\hat{u}^{n}(x, t)-u_{0}^{n}(x)\right) a_{i}(t) d t, & i=1,2, \\
u_{i}(x)=\left(\hat{u}^{a}(x, t)-u_{0}^{a}, a_{i}(t)\right)_{T^{a}}, & i=3,4,
\end{array}
$$

which we additionally orthonormalize. The expressions $u_{0}^{n}$ and $u_{0}^{a}$ are the mean flows $u_{0}^{n}(x)=1 / N \sum_{i=1}^{N} \hat{u}_{i}^{n}(x)$ in the unactuated respectively $u_{0}^{a}(x)=1 / N \sum_{i=1}^{N} \hat{u}_{i}^{a}(x)$ in the actuated case.

Low order dynamical system. The filter modes contain significant information gained from the URANS solution by the $k$ - $\omega$-model. Following [20, 29], the main idea of the ROM is to set up a small size system of ODEs for $\left(a_{1}(t), \cdots, a_{4}(t)\right)^{T}$ that rebuilds the computed fluid flow best under the operating conditions. Similarly to the work of Luchtenburg et al. [20], the dynamical system consists of the uniformly oscillating of the unactuated and the actuated coefficient pairs $\left(a_{1}, a_{2}\right)$ respectively $\left(a_{3}, a_{4}\right)$, which are coupled through the equations for amplified saturation. The dynamical system is set up as follows:

$$
\begin{aligned}
& \dot{a}_{1}=\tilde{\sigma}^{n} a_{1}-\omega^{n} a_{2} \\
& \dot{a}_{2}=\omega^{n} a_{1}+\tilde{\sigma}^{n} a_{2} \\
& \dot{a}_{3}=\tilde{\sigma}^{a} a_{3}-\omega^{a} a_{4}+g_{31} g+g_{32} \dot{g} \\
& \dot{a}_{4}=\omega^{a} a_{3}+\tilde{\sigma}^{a} a_{4}+g_{41} g+g_{42} \dot{g} \\
& \tilde{\sigma}^{n}=\sigma^{n}-\beta_{1}\left(A^{n}\right)^{2}-\beta_{2}\left(A^{a}\right)^{2} \\
& \tilde{\sigma}^{a}=\sigma^{a}
\end{aligned}
$$


with $A^{n}=\sqrt{a_{1}^{2}+a_{2}^{2}}, A^{a}=\sqrt{a_{3}^{2}+a_{4}^{2}}$ and $g=B \cos \left(\omega^{a} t\right)$, where $B$ is the amplitude of the actuation signal and $\omega^{a}$ is the associated phase. Because the snapshots do not carry transient data, we have to select the amplification rates $\sigma^{n}, \sigma^{a}$ as follows:

- $\sigma^{n}=0.15$ is an empirical value, if the cord-length of the wing is 1 . Because the flap is the active part of the configuration, we choose $\sigma^{n}=0.15 \frac{U_{\infty}}{c_{f l}}$.

- $\sigma^{a}=-\frac{1}{T_{\text {con }}}$ where $T_{c o n}$ is the time that one vortex needs to pass the flap-length $c_{f l}$. We read off this value from the snapshots.

If the fluid flow is in the unactuated state, then we should have no energy in the coefficients $a_{3}, a_{4}$, i.e. $a_{3}=a_{4}=0$, hence $A^{a}=0$. Moreover, we require $\tilde{\sigma}^{n}=$ $\sigma^{n}-\beta_{1}\left(A^{n}\right)^{2}=0$ holds for the unactuated flow dynamics. This expresses the fact that there is no additional energy contribution to the natural oscillatory behavior of $a_{1}, a_{2}$. Thus $\beta_{1}$ can be determined by

$$
\beta_{1}=\sigma^{n} \frac{1}{\left(A^{n}\right)^{2}}=\sigma^{n} \frac{1}{\left(r^{n}\right)^{2}}
$$

In the actuated case, the energy of the modes with the natural frequency vanishes. To identify the parameter $\beta_{2}$, we need an additional set of snapshots with a small amplitude of actuation $B$ where the energy $\tilde{A}^{n}$, contained in the associated filtered coefficients $\tilde{a}_{1}, \tilde{a}_{2}$, is not equal to zero. This is to determine the so-called vanishingratio of the energy with respect to increasing $B$. For this actuation amplitude, we compute the filtered coefficients and the associated energies $\left(\tilde{A}^{n}\right)^{2}$ and $\left(\tilde{A}^{a}\right)^{2}$. We determine $\beta_{2}$ by assuming that the energy in this system remains constant over all amplitudes $B$ by

$$
\sigma^{n}=\beta_{1}\left(\tilde{A}^{n}\right)^{2}+\beta_{2}\left(\tilde{A}^{a}\right)^{2}
$$

notice that $\left(\tilde{A}^{n}\right)^{2},\left(\tilde{A}^{a}\right)^{2}$ are associated with $B$.

The dynamical system (9) contains free parameters $g_{31}, g_{32}, g_{41}$ and $g_{42}$ to calibrate the selected actuation to the dynamical system. Remind that the actuation $g$ and its derivative $\dot{g}$ are $g=B \cos \left(\omega^{a} t\right)$ and $\dot{g}=-B \omega^{a} \sin \left(\omega^{a} t\right)$, where the actuation amplitude $B$ is our optimization variable. Therefore, we multiply the third and fourth equation by $g$ and $\dot{g}$, respectively, and integrate over $\left[0, T^{a}\right]$. This eliminates $g_{32}, g_{42}$ and $g_{31}, g_{41}$, respectively. For instance

$$
\left(\dot{a_{3}}, g\right)_{T^{a}}=\sigma_{a}\left(a_{3}, g\right)_{T^{a}}-\omega_{a}\left(a_{4}, g\right)_{T^{a}}+g_{31}(g, g)_{T^{a}}
$$

leads to

$$
g_{31}=\left(\left(\dot{a}_{3}, g\right)_{T^{a}}-\sigma^{a}\left(a_{3}, g\right)_{T^{a}}+\omega^{a}\left(a_{4}, g\right)_{T^{a}}\right) /(g, g)_{T^{a}} .
$$

Note that $(\dot{g}, g)_{T^{a}}$ vanishes in the long term average.

Computation of lift. Based on the dynamical system $\left(\dot{a}_{i}\right)_{i}$, the lift is obtained by the following ansatz with unknown coefficients $c_{i j}$ and $c_{k}$

$$
C_{L}\left(a_{1}, a_{2}, a_{3}, a_{4}\right)=c_{l 0}+\sum_{i=1}^{4} c_{1 i} a_{i}+c_{5}\left(A^{n}\right)^{2}+c_{6}\left(A^{n}\right)^{4}
$$


There is no limitation to the energy $A^{a}$ of the actuated case with respect to increasing $B$, hence $A^{a}$ is not included in (13). The ansatz (13) is motivated by a global momentum balance equation and the constant and linear term in (13) are related to this equation. The lift effect of base-flow variation can be lumped in $c_{5}\left(A^{n}\right)^{2}+c_{6}\left(A^{n}\right)^{4}$ assuming slow transients, see [29] in this volume. The parameters $c_{i j}$ and $c_{k}$ are obtained by a least-squares fit of $C_{L}\left(\left(a_{i}^{n}\right)\right)$ and $C_{L}\left(\left(a_{i}^{a}\right)\right)$ to the original lift values of the URANS simulation. The $\left(a_{i}^{n}\right)$ are the filtered coefficients of the unactuated case and $\left(a_{i}^{a}\right)$ are the filtered coefficients of the actuated case. Our goal is to fit the parameters in the sense that the simulated lift values are reproduced by the lift formula (13) in the unactuated case $C_{L U}$ based on $\left(a_{i}^{n}\right)$ as well as the lift values in the actuated case $C_{L A}$ based on $\left(a_{i}^{a}\right)$. This leads to the problem

$$
\min _{c_{i j}} F\left(c_{i j}\right)=\left\|C_{L A}(\cdot)-C_{L}\left(c_{i j}\right)\left(\left(a_{i}^{a}(\cdot)\right)\right)\right\|^{2}+\left\|C_{L N}(\cdot)-C_{L}\left(c_{i j}\right)\left(\left(a_{i}^{n}(\cdot)\right)\right)\right\|^{2} .
$$

Finally, we have to solve the following optimization problem

$$
\max _{B \in B_{a d}} C_{L}\left(a_{1}, a_{2}, a_{3}, a_{4}\right)
$$

subject to the ODE system (9), where $B_{a d}:=[0, \hat{B}]$ with an upper boundary $\hat{B} \in \mathbb{R}$ and 0 as lower boundary.

Numerical example. A high-lift configuration with observation region $\Omega$ presented in Figure 4 was considered, see [20] for details.

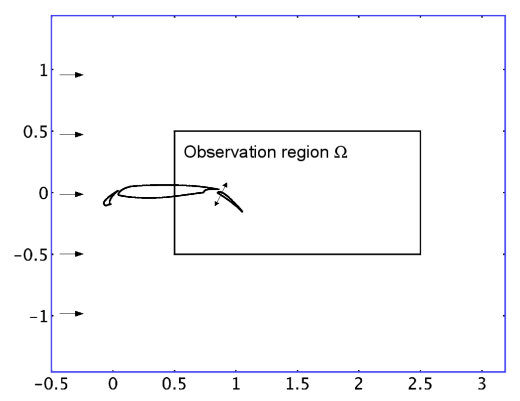

Fig. 4 The SCCH high-lift configuration, where the periodic excitation is implemented on the flap.

The actuation amplitude was $B=3.5888$ and we worked with the parameters

$$
\begin{array}{llrl}
\sigma^{n}=0.5906 & g_{31}=0.0284, g_{32} & =0.0000, & \omega^{n}=5.5407, \\
\sigma^{a}=-2.0042, & g_{41}=0.0000, g_{42} & =-0.0019, & \omega^{a}=14.8412
\end{array}
$$

for $c_{f l}=0.254, \beta_{1}=14.75$ and $\beta_{2}=654.0806$, calculated with an actuation amplitude of $B=1.19$. An example of the phase portraits for the coefficients $a_{1}, \cdots, a_{4}$ of 
the dynamical system (9) is presented in Figure 5.
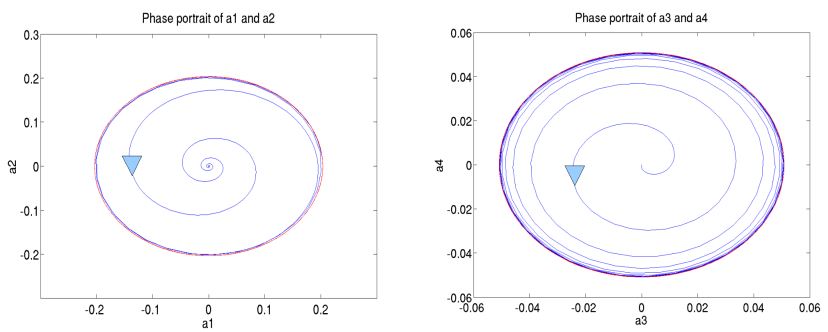

Fig. 5 Phase portraits of $\left(a_{1}, a_{2}\right)$ (left) and $\left(a_{3}, a_{4}\right)$ (right) of system (9) with full actuation, starting with $\left(a_{1}, a_{2}\right)$ in the attractor $r^{n}$ and $\left(a_{3}, a_{4}\right)=(0,0)$.

Calibrating the parameters of the lift formula (13) to this data, we get $c_{l 0}=$ 2.2238, $c_{11}=0.2295, c_{12}=-0.6858, c_{13}=1.6717, c_{14}=-0.2963, c_{5}=-8.3606$, $c_{6}=39.7410$. Figure 6 shows the coincidence of $C_{L}\left(a_{i}\right)$ with the lift-values of the URANS simulation, where $\left(a_{i}\right)$ are the filtered coefficients.
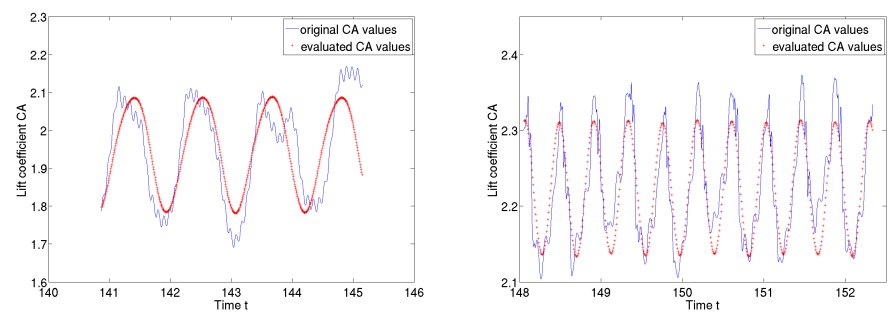

Fig. 6 Comparison of the lift values of the URANS simulation with those obtained by the lift formula based on the filtered coefficients $a_{1}, \cdots, a_{4}$ : Natural flow (left), actuated flow (right).

The mean values differ in both cases not more than $1 \%$. Evaluating $C_{L}$ with the $a_{i}$ 's as the solutions of the dynamical system, once computed with $B=0$ and once with the full actuation $B=3.5888$, we get mean values of around 1.96 respectively 2.24 and the results presented in Figure 7.

Solving this dynamical system with several actuation amplitudes $B=0$ to $B=$ 4.3, we resolve the average lift values presented in Figure 8; for $B=0$ an average lift of 1.96 and for $B=3.5888$ an average lift of 2.20 . 

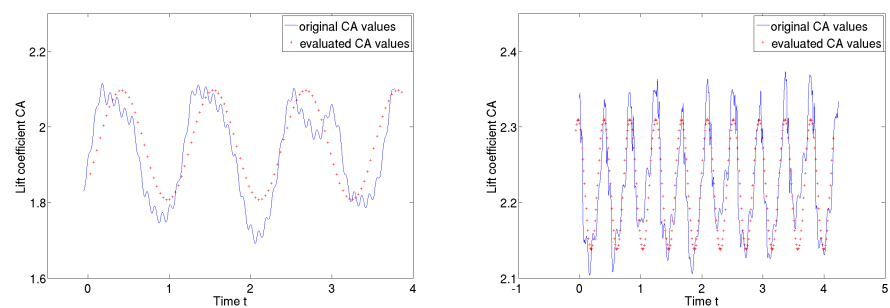

Fig. 7 Comparison of the lift values of the URANS simulation with those obtained by the lift formula based on $a_{1}, \cdots, a_{4}$ of the dynamical system: Natural flow (left), actuated flow (right).

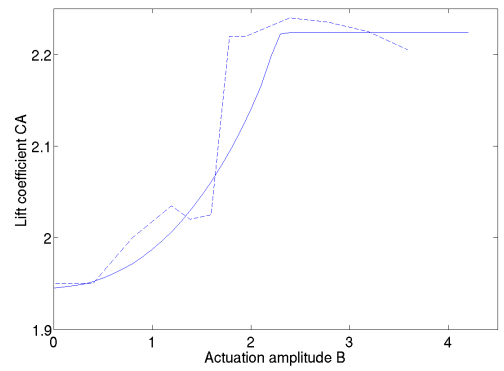

Fig. 8 Comparison of the calculated lift coefficients (continuous lines) with those obtained by the URANS simulation (dashed lines) over the actuation amplitude $B$.

\section{Conclusion}

We considered two settings of high-lift configurations. In the case of steady state Navier-Stokes equations with low Reynolds number, we established first-order necessary optimality conditions for a problem with integral state constraint on the drag. The main theoretical difficulty was the appearance of low regularity controls in a Dirichlet boundary condition. The optimal control is obtained by direct numerical solution of the established optimality system.

An associated nonstationary case with high Reynolds number was investigated by a WILCOX98 turbulence model. To deal with the unavoidable curse of dimension, a robust reduced order model was set up that fits best to snapshots computed by the full system in the natural and the actuated state. The ROM reproduces the nonlinear behavior of the system sufficiently well so that an optimization of periodic actuation will lead to reasonable results.

In particular, the application of trust-region proper orthogonal decomposition (TRPOD) should be considered to set up an improved reduced-order model. In [4] 
a ROM was used to minimize the total mean drag for a circular cylinder wake flow by updating the ROM during a (TRPOD) approach, we refer also to [3].

Acknowledgements We acknowledge stimulating discussions with A. Carnarius, B. Günther, D. M. Luchtenburg, M. Nestler, and F.Thiele. We are very grateful to the group "Computational Fluid Dynamics and Aeroacoustics", Institute of Fluid Mechanics and Engineering Acoustics, TU Berlin, chaired by F. Thiele, for providing us with associated snapshots. The authors acknowledge the excellent working conditions and the support by the DFG funded Collaborative Research Center Sfb 557 "Control of Complex Turbulent Shear Flow" at TU Berlin.

\section{References}

1. F. Abergel and R. Temam. On some control problems in fluid mechanics. Theoret. Comput. Fluid Dynam., 1:303-325, 1990.

2. K. Afanasiev and M. Hinze. Adaptive control of a wake flow using proper orthogonal decomposition. In Lecture Notes in Pure and Applied Mathematics 216, 317-332, Shape Optimization and Optimal Design, Marcel Dekker.

3. E. Arian, M. Fahl, and E.W. Sachs. Trust-region proper-orthogonal decomposition for flow control. Technical Report 2000-25, Universität Trier, 2000.

4. M. Bergmann and L. Cordier. Optimal control of the cylinder wake in the laminar regime by trust-region methods and pod reduced-order models. J. Comput. Phys., 227(16):7813-7840, 2008.

5. T. Bewley, P. Moin, and R. Temam. DNS-based predictive control of turbulence: an optimal benchmark for feedback algorithms. J. Fluid Mech., 447:179-225, 2001.

6. A. Carnarius, B. Günther, F. Thiele, D. Wachsmuth, F. Tröltzsch, and J.C. Reyes. Numerical study of the optimization of separation control. In Proceedings of the 45th AIAA Aerospace Sciences Meeting and Exhibit, Reno, 8-11 January 2007, AIAA 2007-58.

7. E. Casas, M. Mateos, and J.-P. Raymond. Error estimates for the numerical approximation of a distributed control problem for the steady-state Navier-Stokes equations. SIAM J. Control Optim., 46(3):952-982, 2007.

8. Ghayour K. Heinkenschloss M. Ulbrich M. Collis, S.S. and S. Ulbrich. Optimal control of unsteady compressible viscous flows. Int. J. Numer. Meth. Fluids, 40, 2002.

9. J. C. de los Reyes and K. Kunisch. A semi-smooth Newton method for control constrained boundary optimal control of the Navier-Stokes equations. Nonlinear Anal., 62(7):1289-1316, 2005.

10. R. Griesse and J. C. de los Reyes. State-constrained optimal control of the three-dimensional stationary Navier-Stokes equations. J. Math. Anal. Appl., 343(1):257-272, 2008.

11. M. D. Gunzburger, L. S. Hou, and Th. P. Svobodny. Analysis and finite element approximation of optimal control problems for the stationary Navier-Stokes equations with Dirichlet controls. RAIRO Modél. Math. Anal. Numér., 25(6):711-748, 1991.

12. M. D. Gunzburger and S. Manservisi. The velocity tracking problem for Navier-Stokes flows with boundary control. SIAM J. Control Optim., 39:594-634, 2000.

13. Max D. Gunzburger. Perspectives in Flow Control and Optimization. Society for Industrial and Applied Mathematics, Philadelphia, PA, USA, 2002.

14. M. Hinze and K. Kunisch. Second-order methods for optimal control of time-dependent fluid flow. SICON, 40, 2001

15. P. Holmes, J. L Lumley, and G. Berkooz. Turbulence, Coherent Structures, Dynamical Systems and Symmetry. Cambridge University Press, Cambridge, 1998.

16. C. John and D. Wachsmuth. Optimal Dirichlet boundary control of Navier-Stokes equations with state constraint. accepted by Numerical Functional Analysis and Optimization, 2009. 
17. B.H. Jørgensen, J.N. Sørensen, and M. Brøns. Low-dimensional modelling of a driven cavity flow with two free parameters. Theoret. Comput. Fluid Dynamics, 16:299-317, 2003.

18. K. Kunisch and S. Volkwein. Galerkin proper orthogonal decomposition methods for a general equation in fluid dynamics. SIAM Journal on Numerical Analysis, 40:492-515, 2002.

19. K. Kunisch and L. Xie. Suboptimal feedback control of flow separation by POD model reduction. SIAM, 2006.

20. D.M. Luchtenburg, B. Günther, B.R. Noack, R. King, and G. Tadmor. A generalized meanfield model of the natural and high-frequency actuated flow around a high-lift configuration. J. Fluid Mech., 623:283-316, 2009.

21. I. Neitzel, U. Prüfert, and T. Slawig. Strategies for time-dependent pde control with inequality constraints using an integrated modeling and simulation environment. Numerical Algorithms, 50(3):241269, 2009.

22. B.R. Noack, K. Afanasiev, M. Morzyński, G Tadmor, and F. Thiele. A hierarchy of lowdimensional models for the transient and post-transient cylinder wake. J. Fluid Mech., 497:335-363, 2008.

23. J.-P. Raymond. Stokes and Navier-Stokes equations with nonhomogeneous boundary conditions. Ann. Inst. H. Poincaré Anal. Non Linéaire, 24(6):921-951, 2007.

24. J. C. de los Reyes and K. Kunisch. A semi-smooth Newton method for regularized stateconstrained optimal control of the Navier-Stokes equations. Computing, 78(4):287-309, 2006.

25. J. C. de los Reyes and F. Tröltzsch. Optimal control of the stationary Navier-Stokes equations with mixed control-state constraints. SIAM J. Control Optim., 46(2):604-629, 2007.

26. Günther B. Schatz, M. and F. Thiele. Computational investigation of separation control for high-lift airfoil flows. Active Flow Control, Springer, 2006.

27. M. Schatz and F. Thiele. Numerical Study of High-Lift Flow with Separation Control by Periodic Excitation. AIAA paper, 2001-0296, 2001.

28. M. Schatz, F. Thiele, R. Petz, and W. Nitsche. Separation control by periodic excitation and its application to a high-lift configuration. AIAA, (2507), 2004.

29. Luchtenburg D.M. Noack B.R. Aleksic K. Pastoor M. King R. Schlegel, M. and G Tadmor. Turbulence control based on reduced-order models. $A F C$, page submitted.

30. S.G. Siegel, J. Seidel, C. Fagley, D.M. Luchtenburg, K. Cohen, and T. McLaughlin. Lowdimensional modelling of a transient cylinder wake using double proper orthogonal decomposition. J. Fluid Mech., 610:1-42, 2008.

31. F.H. Tinapp and W. Nitsche. On active control of high-lift flow. In In W. Rodi and D. Laurence, editors, Proc. 4th Int. Symposium on Engineering Turbulence Modelling and Measurements, Corsica, Elsevier Science, 1999.

32. F. Tröltzsch and D. Wachsmuth. Second-order sufficient optimality conditions for the optimal control of Navier-Stokes equations. ESAIM: COCV, 12:93-119, 2006.

33. D. Wachsmuth. Sufficient second-order optimality conditions for convex control constraints. J. Math. Anal. App., 319:228-247, 2006.

34. D. Wachsmuth. Analysis of the SQP-method for optimal control problems governed by the instationary Navier-Stokes equations based on $L^{p}$-theory. SIAM Journal Control and Optimization, 46:1133-1153, 2007.

35. Y. Wang, F. Tröltzsch, and G. Bärwolff. The POD Dirichlet Boundary Control of the NavierStokes Equations: A Low-dimensional Approach to Optimal Control with High Smoothness. Technical Report 23-2009, Technische Universität Berlin, 2009.

36. E. Wassen and F. Thiele. Active control of a model vehicle wake. Journal of Turbulence, 2006.

37. D.C. Wilcox. Simulation of Transition with a Two-Equation Turbulence Model. AIAA JOURNAL, 32, 1994. 